\title{
ASYMPTOTIC PROPERTY OF SOLUTIONS OF A CLASS OF THIRD-ORDER DIFFERENTIAL EQUATIONS
}

\author{
N. PARHI AND P. DAS
}

(Communicated by Kenneth R. Meyer)

\begin{abstract}
It has been shown that the equation
\end{abstract}
(*)

$$
y^{\prime \prime \prime}+a(t) y^{\prime \prime}+b(t) y^{\prime}+c(t) y=0,
$$

where $a, b$, and $c$ are real-valued continuous functions on $[\alpha, \infty)$ such that $a(t) \geq 0, b(t) \leq 0$, and $c(t)>0$, admits at most one solution $y(t)$ (neglecting linear dependence) with the property $y(t) y^{\prime}(t)<0, y(t) y^{\prime \prime}(t)>0$ for $t \in[\alpha, \infty)$ and $\lim _{t \rightarrow \infty} y(t)=0$, if $(*)$ has an oscillatory solution. Further, sufficient conditions have been obtained so that $(*)$ admits an oscillatory solution.

\section{In this paper, we consider}

$$
y^{\prime \prime \prime}+a(t) y^{\prime \prime}+b(t) y^{\prime}+c(t) y=0,
$$

where $a, b$, and $c \in C([\alpha, \infty), R), \alpha \in R$, such that $a(t) \geq 0, b(t) \leq 0$, and $c(t)>0$ for $t \in[\alpha, \infty)$. A solution of $(1)$ is said to be oscillatory if it has arbitrarily large zeros and is said to be nonoscillatory otherwise.

The purpose of this work is to show that (1) admits at most one solution $y(t)$ (neglecting linear dependence) with the property $y(t) y^{\prime}(t)<0, y(t) y^{\prime \prime}(t)>0$ for $t \in[\alpha, \infty)$ and $\lim _{t \rightarrow \infty} y(t)=0$, if (1) has an oscillatory solution. Jones has obtained this result for (1) with $a(t) \equiv 0$ (see [3, 4]). In his work [1], Erbe has partially generalized the work of Jones to (1). Besides other conditions, he has assumed that $a(t) \leq 0$. Moreover, our conditions are comparatively simpler than those of Erbe. We are thankful to Prof. M. Gregus for bringing this problem to our attention.

Suppose that $a(t), b(t)$, and $c(t)$ in (1) are constants $a, b$, and $c$, respectively. It is well known that (1) admits an oscillatory solution if

$$
\frac{2 a^{3}}{27}-\frac{a b}{3}+c-\frac{2}{3 \sqrt{3}}\left(\frac{a^{2}}{3}-b\right)^{3 / 2}>0 \text {. }
$$

Received by the editors May 13, 1989 and, in revised form, August 21, 1989.

1980 Mathematics Subject Classification (1985 Revision). Primary 34C10, 34C11.

The work of the second author was done under a scheme supported by the University Grants Commission, New Delhi under grant No. F.8-9/87 (SR-III). 
In this paper we have obtained a result which generalizes the above result to variable coefficients. Lazer's result [5, Theorem 1.3] follows from our result if $a(t) \equiv 0$.

2. This section deals with asymptotic behavior of nonoscillatory solutions of (1) in the presence of an oscillatory solution. Following two lemmas are easy generalizations of Lazer's results (see [5]).

Lemma 1. If $y(t)$ is a solution of (1) satisfying $y(\beta) \geq 0, y^{\prime}(\beta) \leq 0, y^{\prime \prime}(\beta)>$ $0, \beta \in(\alpha, \infty)$, then $y(t)>0, y^{\prime}(t)<0, y^{\prime \prime}(t)>0$, and $y^{\prime \prime \prime}(t) \leq 0$ for $t \in[\alpha, \beta)$.

Lemma 2. Equation (1) admits a nonoscillatory solution $y(t)$ such that $y(t)>0$, $y^{\prime}(t)<0, y^{\prime \prime}(t)>0, y^{\prime \prime \prime}(t)<0$ for $t \in[\alpha, \infty)$ and $\lim _{t \rightarrow \infty} y^{\prime}(t)=0=$ $\lim _{t \rightarrow \infty} y^{\prime \prime}(t), \lim _{t \rightarrow \infty} y(t)=k \neq \pm \infty$.

In the following we state a lemma due to Erbe [1] for our use in the sequel.

Lemma 3. A necessary and sufficient condition for (1) to have an oscillatory solution is that for any nontrivial nonoscillatory solution $u(t)$ of (1) the following conditions hold:

$$
u(t) u^{\prime}(t) u^{\prime \prime}(t) u^{\prime \prime \prime}(t) \neq 0, \quad \operatorname{sgn} u(t)=\operatorname{sgn} u^{\prime \prime}(t) \neq \operatorname{sgn} u^{\prime}(t)=\operatorname{sgn} u^{\prime \prime \prime}(t)
$$

for $t \geq \alpha$ and

$$
\lim _{t \rightarrow \infty} u^{\prime}(t)=\lim _{t \rightarrow \infty} u^{\prime \prime}(t)=0, \quad \lim _{t \rightarrow \infty} u(t)=\lambda \neq \pm \infty .
$$

Theorem 4. Suppose that (1) has an oscillatory solution and $\lim _{t \rightarrow \infty} t^{2} b(t) \neq 0$. If $u(t)$ is a nonoscillatory solution of $(1)$, then $\lim _{t \rightarrow \infty} u(t)=0$.

Proof. Without any loss of generality, we may assume that $u(t)>0$ for $t \geq \alpha$. From Lemma 3 we obtain $u^{\prime}(t)<0, u^{\prime \prime}(t)>0, u^{\prime \prime \prime}(t)<0$ for $t \geq \alpha$ and $\lim _{t \rightarrow \infty} u^{\prime}(t)=0=\lim _{t \rightarrow \infty} u^{\prime \prime}(t), \lim _{t \rightarrow \infty} u(t)=\lambda$, where $0 \leq \lambda<\infty$. If possible, let $\lambda \neq 0$. Further, without any loss of generality we may assume that $\lambda=\frac{1}{2}$. Since $u^{\prime}(t)<0, t \geq \alpha$, it is possible to find $t_{0}>\alpha$ such that $\frac{1}{2}<u(t)<1$ for $t \geq t_{0}$.

Equation (1) may be written as

$$
\left(r(t) u^{\prime \prime}(t)\right)^{\prime}+q(t) u^{\prime}(t)+p(t) u(t)=0,
$$

where $r(t)=\exp \left(\int_{\alpha}^{t} a(s) d s\right), p(t)=c(t) r(t)$, and $q(t)=b(t) r(t)$. If $v(t)$ is the oscillatory solution of (1), then $W(u, v)(t)=u(t) v^{\prime}(t)-u^{\prime}(t) v(t)$ is an oscillatory solution of

$$
\left(\frac{r(t) x^{\prime}}{u(t)}\right)^{\prime}+\left(\frac{r(t) u^{\prime \prime}(t)+q(t) u(t)}{u^{2}(t)}\right) x=0 .
$$

Proceeding as in Jones [3], one may get $\lim _{t \rightarrow \infty} t^{2} u^{\prime \prime}(t)=0$. Since $r(t)$ is monotonic increasing, we have

$$
\frac{r(t)}{u(t)}>r(t)>r(\alpha)=1, \quad \text { for } t \geq t_{0} .
$$


Further,

implies that

$$
\lim _{t \rightarrow \infty} t^{2}\left[u^{\prime \prime}(t)+b(t) u(t)\right]<0
$$

$$
\lim _{t \rightarrow \infty} t^{2}\left[r(t) u^{\prime \prime}(t)+q(t) u(t)\right]=\lim _{t \rightarrow \infty} t^{2} r(t)\left[u^{\prime \prime}(t)+b(t) u(t)\right]<0 .
$$

Hence the equation

$$
z^{\prime \prime}+\left[\frac{r(t) u^{\prime \prime}(t)+q(t) u(t)}{u^{2}(t)}\right] z=0
$$

is nonoscillatory. From Sturm's comparison theorem it follows that (3) is nonoscillatory, a contradiction.

Hence the theorem is proved.

Remark. The condition $\lim _{t \rightarrow \infty} t^{2} b(t) \neq 0$ in Theorem 4 may be replaced by the assumption $r(t)$ is bounded.

Theorem 5. Suppose that (1) has an oscillatory solution and $\int_{\alpha}^{\infty} c(t) d t=\infty$. If $u(t)$ is a nonoscillatory solution of $(1)$, then $\lim _{t \rightarrow \infty} u(t)=0$.

Proof. Let $u(t)>0$ for $t \geq \alpha$. From Lemma 3, we get $u^{\prime}(t)<0, u^{\prime \prime}(t)>0$, $u^{\prime \prime \prime}(t)<0$ for $t \geq \alpha$ and $\lim _{t \rightarrow \infty} u^{\prime}(t)=0=\lim _{t \rightarrow \infty} u^{\prime \prime}(t), \lim _{t \rightarrow \infty} u(t)=\lambda$, where $0 \leq \lambda<\infty$. If possible, let $\lambda>0$. Now integrating (2) from $\alpha$ to $t$, we obtain

$$
\begin{aligned}
r(t) u^{\prime \prime}(t) & =u^{\prime \prime}(\alpha)-\int_{\alpha}^{t} q(s) u^{\prime}(s) d s-\int_{\alpha}^{t} p(s) u(s) d s \\
& <u^{\prime \prime}(\alpha)-u(t) \int_{\alpha}^{t} r(s) c(s) d s \\
& <u^{\prime \prime}(\alpha)-u(t) \int_{\alpha}^{t} c(s) d s .
\end{aligned}
$$

This in turn implies that $u^{\prime \prime}(t)<0$ for large $t$, a contradiction.

This completes the proof of the theorem.

Remark. From Lemma 2 it follows that if the hypotheses of either Theorem 4 or 5 are satisfied, then equation (1) admits a nonoscillatory solution which tends to zero. From the next theorem it follows that every other nonoscillatory solution of (1) is a constant multiple of this solution.

Theorem 6. Suppose that (1) admits an oscillatory solution. If $y(t)$ is a nonoscillatory solution of $(1)$ with $\lim _{t \rightarrow \infty} y(t)=0$, then every nonoscillatory solution of (1) is a constant multiple of $y(t)$.

Proof. Equation (1) may be written as

$$
\left(r(t) y^{\prime \prime}\right)^{\prime}+q(t) y^{\prime}+p(t) y=0
$$

where $r(t)=\exp \left(\int_{\alpha}^{t} a(s) d s\right), p(t)=c(t) r(t)$, and $q(t)=b(t) r(t)$. Let $u_{1}(t)$ and $u_{2}(t)$ be two solutions of $(4)$ on $[\alpha, \infty)$ with initial conditions

$$
u_{1}(\alpha)=u_{1}^{\prime}(\alpha)=0, \quad r(\alpha) u_{1}^{\prime \prime}(\alpha)=1
$$


and

$$
u_{2}(\alpha)=u_{2}^{\prime \prime}(\alpha)=0, \quad u_{2}^{\prime}(\alpha)=-1
$$

From Lemma 1 of this paper and Theorem 3.4 due to Hanan [2], it follows that both $u_{1}(t)$ and $u_{2}(t)$ are oscillatory solutions of (4).

Clearly, $W_{1}(t) \equiv W\left(u_{1}, u_{2}\right)(t)=u_{1}(t) u_{2}^{\prime}(t)-u_{1}^{\prime}(t) u_{2}(t)$ is a solution of the adjoint equation

$$
\left(r(t) y^{\prime}\right)^{\prime \prime}+(q(t) y)^{\prime}-p(t) y=0
$$

with $W_{1}(\alpha)=W_{1}^{\prime}(\alpha)=0$ and $\left(r W_{1}^{\prime}\right)^{\prime}(\alpha)>0$.

It is easy to see that $W_{1}(t)>0$ for $t>\alpha$. Indeed, $\left(r W_{1}^{\prime}\right)^{\prime}(\alpha)>0$ and $\left(r W_{1}^{\prime}\right)^{\prime}(t)$ continuous imply that $\left(r W_{1}^{\prime}\right)^{\prime}(t)>0, t \in[\alpha, \alpha+\delta)$, for some $\delta>0$. This in turn implies that $W_{1}^{\prime}(t)>0$ for $t \in(\alpha, \alpha+\delta)$ and hence $W_{1}(t)>0$ for $t \in(\alpha, \alpha+\delta)$. We claim that $W_{1}(t)>0$ for $t>\alpha$. If not, there is a $\beta>\alpha$ such that $W_{1}(\beta)=0$ and $W_{1}(t)>0$ for $t \in(\alpha, \beta)$. Since $W_{1}(t)$ is a solution of $(5),\left(r W_{1}^{\prime}\right)^{\prime}(t)+q(t) W_{1}(t)$ is nondecreasing in $[\alpha, \beta)$. Hence $\left(r W_{1}^{\prime}\right)^{\prime}(t)>0$ for $t \in[\alpha, \beta]$. Consequently, $W_{1}^{\prime}(t)>0$ for $t \in(\alpha, \beta]$, a contradiction. Hence our claim holds, Further, $u_{1}(t)$ and $u_{2}(t)$ are linearly independent oscillatory solutions of the second-order differential equation

$$
\left|\begin{array}{lll}
u_{1}(t) & u_{2}(t) & x \\
u_{1}^{\prime}(t) & u_{2}^{\prime}(t) & x^{\prime} \\
u_{1}^{\prime \prime}(t) & u_{2}^{\prime \prime}(t) & x^{\prime \prime}
\end{array}\right|=0
$$

that is,

$$
\left(\frac{x^{\prime}}{W_{1}(t)}\right)^{\prime}+\left(\frac{\left(r W_{1}^{\prime}\right)^{\prime}(t)+q(t) W_{1}(t)}{r(t) W_{1}^{2}(t)}\right) x=0 .
$$

So any nontrivial linear combination of $u_{1}(t)$ and $u_{2}(t)$ is oscillatory.

Clearly, $\left\{u_{1}(t), u_{2}(t), y(t)\right\}$ forms a basis of solution space of (4).

Without any loss of generality, we may assume $y(t)>0$ for $t \geq t_{0}^{*} \geq \alpha$.

If possible, let $w(t)$ be a nonoscillatory solution of $(1)$ on $[\alpha, \infty)$ such that $w(t)$ and $y(t)$ are linearly independent. So $w(t)=\lambda_{1} u_{1}(t)+\lambda_{2} u_{2}(t)+\lambda_{3} y(t)$, where $\lambda_{1}, \lambda_{2}, \lambda_{3}$ are constants. $\lambda_{3}=0$ implies that $w(t)$ is oscillatory. So $\lambda_{3} \neq 0$. Dividing $\lambda_{3}$, we get $z(t)=y(t)+c_{1} u_{1}(t)+c_{2} u_{2}(t)$, where $z(t)=$ $w(t) / \lambda_{3}, c_{1}=\lambda_{1} / \lambda_{3}$ and $c_{2}=\lambda_{2} / \lambda_{3}$. Clearly, $c_{1}$ and $c_{2}$ cannot be equal to zero simultaneously. Since $c_{1} u_{1}(t)+c_{2} u_{2}(t)$ is oscillatory, then $y(t)$ and $z(t)$ are of the same sign for $t \geq t_{0} \geq t_{0}^{*} \geq \alpha$.

Setting $y_{1}(t)=-c_{1} u_{1}(t)-c_{2} u_{2}(t)$, we obtain $z(t)=y(t)-y_{1}(t)$. Clearly, $y_{1}(t)$ is an oscillatory solution of $(6)$ and (4). Let $t_{1}>t_{0}$ be a zero of $y_{1}(t)$ such that $y_{1}^{\prime}\left(t_{1}\right)>0$. Let $y_{2}(t)$ be a solution of $(4)$ on $\left[t_{1}, \infty\right)$ such that $y_{2}\left(t_{1}\right)=$ $y_{2}^{\prime}\left(t_{1}\right)=0$ and $y_{2}^{\prime \prime}\left(t_{1}\right)=1$. From Hanan's result [2, Theorem 3.4] it follows that $y_{2}(t)$ is oscillatory. Clearly, $W(t) \equiv W\left(y_{1}, y_{2}\right)(t)=y_{1}(t) y_{2}^{\prime}(t)-y_{1}^{\prime}(t) y_{2}(t)$ is a solution of (5) with $W\left(t_{1}\right)=0=W^{\prime}\left(t_{1}\right)$ and $\left(r W^{\prime}\right)^{\prime}\left(t_{1}\right)>0$. Hence $W(t)>0$ 
for $t>t_{1}$. Consequently, it follows from (5) that $\left(r W^{\prime}\right)^{\prime}+q W$ is increasing in $\left[t_{1}, \infty\right)$. So, for $t>t_{1}$,

$$
\left(r W^{\prime}\right)^{\prime}(t) \geq\left(r W^{\prime}\right)^{\prime}(t)+q(t) W(t)>\left(r W^{\prime}\right)^{\prime}\left(t_{1}\right)>0 .
$$

This in turn implies that $W^{\prime}(t)>0$ for $t>t_{1}$. Clearly, $\left\{y_{1}(t), y_{2}(t), y(t)\right\}$ forms a basis of solution space of (4), because $y_{1}(t)$ and $y_{2}(t)$ are linearly independent oscillatory solutions of the second-order differential equation.

$$
\left(\frac{x^{\prime}}{W(t)}\right)^{\prime}+\left(\frac{\left(r W^{\prime}\right)^{\prime}(t)+q(t) W(t)}{r(t) W^{2}(t)}\right) x=0 .
$$

So

a nonzero constant, that is,

$$
\left|\begin{array}{lll}
y_{1}(t) & y_{2}(t) & y(t) \\
y_{1}^{\prime}(t) & y_{2}^{\prime}(t) & y^{\prime}(t) \\
r(t) y_{1}^{\prime \prime}(t) & r(t) y_{2}^{\prime \prime}(t) & r(t) y^{\prime \prime}(t)
\end{array}\right|=k,
$$

$$
k=W(t) r(t) y^{\prime \prime}(t)-W^{\prime}(t) r(t) y^{\prime}(t)+\left(\left(r W^{\prime}\right)^{\prime}(t)+q(t) W(t)\right) y(t) .
$$

From Lemma 3 it follows that $y^{\prime}(t)<0$ and $y^{\prime \prime}(t)>0, t>t_{1}$. So (7) yields that $k>0$ and, for $t>t_{1}$,

$$
0<\left(\left(r W^{\prime}\right)^{\prime}(t)+q(t) W(t)\right) y(t)<k .
$$

Let $\left\{\sigma_{n}\right\}$ be an increasing sequence of maximum points of $y_{1}(t)$ such that $\sigma_{n}>t_{1}$. So, $y_{1}\left(\sigma_{n}\right)>0$ and $y_{1}^{\prime}\left(\sigma_{n}\right)=0$. Since $z(t)>0$ for $t \geq t_{0}$, it follows from (7) that

$$
\begin{aligned}
0 & \leq\left[\left(r W^{\prime}\right)^{\prime}\left(\sigma_{n}\right)+q\left(\sigma_{n}\right) W\left(\sigma_{n}\right)\right] y_{1}\left(\sigma_{n}\right) \\
& <\left[\left(r W^{\prime}\right)^{\prime}\left(\sigma_{n}\right)+q\left(\sigma_{n}\right) W\left(\sigma_{n}\right)\right] y\left(\sigma_{n}\right)<k .
\end{aligned}
$$

Further, since $\lim _{n \rightarrow \infty} y\left(\sigma_{n}\right)=0$, then $\lim _{n \rightarrow \infty} y_{1}\left(\sigma_{n}\right)=0$. Consequently,

$$
\lim _{n \rightarrow \infty}\left[\left(r W^{\prime}\right)^{\prime}\left(\sigma_{n}\right)+q\left(\sigma_{n}\right) W\left(\sigma_{n}\right)\right] y_{1}^{2}\left(\sigma_{n}\right)=0
$$

On the other hand, if

$$
H(t)=r(t) W(t)\left(y_{1}^{\prime}(t)\right)^{2}+\left(\left(r W^{\prime}\right)^{\prime}(t)+q(t) W(t)\right) y_{1}^{2}(t),
$$

then

$$
\begin{aligned}
H^{\prime}(t)= & 2 r(t) W(t) y_{1}^{\prime}(t) y_{1}^{\prime \prime}(t)+(r W)^{\prime}(t)\left(y_{1}^{\prime}(t)\right)^{2} \\
& +p(t) W(t) y_{1}^{2}(t)+2\left(\left(r W^{\prime}\right)^{\prime}(t)+q(t) W(t)\right) y_{1}(t) y_{1}^{\prime}(t) .
\end{aligned}
$$

Since $y_{1}(t)$ is a solution of $(8)$, we have

$$
r(t) W(t) y_{1}^{\prime \prime}(t)=r(t) W^{\prime}(t) y_{1}^{\prime}(t)-\left[\left(r W^{\prime}\right)^{\prime}(t)+q(t) W(t)\right] y_{1}(t)
$$

and hence, for $t>t_{1}$,

$$
H^{\prime}(t)=2 r(t) W^{\prime}(t)\left(y_{1}^{\prime}(t)\right)^{2}+(r W)^{\prime}(t)\left(y_{1}^{\prime}(t)\right)^{2}+p(t) W(t) y_{1}^{2}(t)>0,
$$


because $(r W)^{\prime}(t)=r^{\prime}(t) W(t)+r(t) W^{\prime}(t)>0$. So, $H(t)$ is a positive increasing function. But from (9) we obtain $\lim _{n \rightarrow \infty} H\left(\sigma_{n}\right)=0$, a contradiction. So $y(t)$ and $z(t)$ are linearly dependent. Consequently, $y(t)$ and $w(t)$ are linearly dependent.

Hence the theorem is proved.

3. In this section we obtain sufficient conditions for the existence of an oscillatory solution of (1). The following lemma due to Erbe [1] is needed in the next theorem.

Lemma 7. If $y(t)$ is a nonoscillatory solution of (1), then there exists a $t_{0} \in$ $[\alpha, \infty)$ such that either

$$
y(t) y^{\prime}(t) \leq 0 \quad \text { for } t \geq t_{0}
$$

or

$$
y(t) y^{\prime}(t) \geq 0 \quad \text { for } t \geq t_{0} .
$$

Further, if (10) holds, then $y(t) y^{\prime}(t) y^{\prime \prime}(t) \neq 0, \operatorname{sgn} y(t)=\operatorname{sgn} y^{\prime \prime}(t) \neq \operatorname{sgn} y^{\prime}(t)$, for $t \geq \alpha$ and $\lim _{t \rightarrow \infty} y^{\prime}(t)=\lim _{t \rightarrow \infty} y^{\prime \prime}(t)=0, \lim _{t \rightarrow \infty} y(t)=\lambda \neq \pm \infty$.

Theorem 8. Suppose that $a(t) \geq 0$ and $a^{\prime}(t) \leq 0$. If

$$
\int^{\infty}\left[\frac{2 a^{3}(t)}{27}-\frac{a(t) b(t)}{3}+c(t)-\frac{2}{3 \sqrt{3}}\left(\frac{a^{2}(t)}{3}-b(t)\right)^{3 / 2}\right] d t=\infty
$$

then (1) admits an oscillatory solution.

Proof. Let $y(t)$ be a nonoscillatory solution of (1). From Lemma 7 it follows that there exists a $t_{0} \in[\alpha, \infty)$ such that (10) holds or (11) holds. In view of Lemma 3 and the second part of Lemma 7 , it is sufficient to prove that (11) does not hold.

Suppose that (11) holds. Setting $u(t)=y^{\prime}(t) / y(t) \geq 0, t \geq t_{0}$, we see that $u(t)$ is a solution of the second-order Riccati equation

$$
z^{\prime \prime}+3 z z^{\prime}+a(t) z^{\prime}=-F(u(t), t),
$$

where $F(u(t), t)=u^{3}(t)+a(t) u^{2}(t)+b(t) u(t)+c(t)$. Clearly, $F(u(t), t)$ attains minimum value for $u(t) \geq 0$ at

$$
u(t)=\frac{1}{3}\left[-a(t)+\left(a^{2}(t)-3 b(t)\right)^{1 / 2}\right] .
$$

So

$$
\begin{aligned}
u^{\prime \prime}(t) & +3 u(t) u^{\prime}(t)+a(t) u^{\prime}(t) \\
& \leq-\left[\frac{2 a^{3}(t)}{27}-\frac{a(t) b(t)}{3}+c(t)-\frac{2}{3 \sqrt{3}}\left(\frac{a^{2}(t)-3 b(t)}{3}\right)^{3 / 2}\right] .
\end{aligned}
$$


Integrating the above inequality from $t_{0}$ to $t$, we obtain

$$
\begin{aligned}
u^{\prime}(t) \leq & u^{\prime}\left(t_{0}\right)+\frac{3}{2} u^{2}\left(t_{0}\right)+a\left(t_{0}\right) u\left(t_{0}\right) \\
& -\int_{t_{0}}^{t}\left[\frac{2 a^{3}(t)}{27}-\frac{a(t) b(t)}{3}+c(t)-\frac{2}{3 \sqrt{3}}\left(\frac{a^{2}(t)-3 b(t)}{3}\right)^{3 / 2}\right] d t .
\end{aligned}
$$

This in turn implies that $\lim _{t \rightarrow \infty} u^{\prime}(t)=-\infty$. Hence $u(t)<0$ for large $t$, a contradiction.

This completes the proof of the theorem.

Each of the following three examples admits only one nonoscillatory solution (neglecting linear dependence) which goes to zero as $t$ goes to infinity.

Example 1. Consider

$$
y^{\prime \prime \prime}+\frac{3}{t} y^{\prime \prime}-\frac{1}{t^{2}} y+e^{t} y=0, \quad t \geq 1 .
$$

This example satisfies conditions of Theorems 5,6 , and 8 .

Example 2. Consider

$$
y^{\prime \prime \prime}+\frac{1}{t^{2}} y^{\prime \prime}-\frac{1}{t^{3}} y^{\prime}+e^{t} y=0, \quad t \geq 1
$$

This example satisfies conditions of Theorems 5 and 8. However, Theorem 6 cannot be applied to this example.

Example 3. Consider

$$
y^{\prime \prime \prime}+\frac{1}{2 t^{1 / 3}} y^{\prime \prime}-\frac{1}{4 t^{2 / 3}} y^{\prime}+\frac{1}{t^{2}} y=0, \quad t \geq 1 .
$$

By Theorem 8, Equation (13) admits an oscillatory solution. This example satisfies conditions of Theorem 6 but not of Theorem 5 .

\section{ACKNOWLEDGMENT}

The authors are thankful to the referee for many helpful remarks.

\section{REFERENCES}

1. L. Erbe, Existence of oscillatory solutions and asymptotic behavior for a class of third order linear differential equations, Pacific J. Math. 64 (1976), 369-385.

2. M. Hanan, Oscillation criteria for third order linear differential equations, Pacific J. Math. 11 (1961), 919-944.

3. G. D. Jones, An asymptotic property of solutions of $y^{\prime \prime \prime}+p y^{\prime}+q y=0$, Pacific J. Math. 48 (1973), 135-138.

4. (1974), 133-136.

5. A. C. Lazer, The behaviour of solutions of the differential equation $y^{\prime \prime \prime}+p(x) y^{\prime}+q(x) y=0$, Pacific J. Math. 17 (1966), 435-466.

6. C. A. Swanson, Comparison and oscillation theory of linear differential equations, Academic Press, New York, 1968.

Department of Mathematics, Berhampur University, Berhampur 760 007, India 